\title{
Editorial
}

\section{Institutional journals as an alternative model for open access}

Open access together with advances in information and communication technology (ICT) have transformed the economics of academic publishing. They have also highlighted a revitalized role for institutional journals. These journals often published through institutional mandates can offer open access without the perceived conflicts of interest created by charging publishing fees. The Memórias do Instituto Oswaldo Cruz provides an interesting case study for other institutional journals.

Traditionally journals were published in printed format and financed through subscriptions to libraries and individuals. Capital was required, to finance the editorial office and the production and publishing costs, until a level of subscription income was reached that made the journal self-financing or profitable. Some journals never obtained sufficient income and required continued subsidies to operate. This need for capital or subsidies meant that journal publishing was generally the preserve of commercial publishers, scientific societies or academic institutions. Very occasionally a brave entrepreneur would enter the field and if successful start their own publishing house.

Advances in ICT revolutionised the journal publishing workflow. Increasingly sophisticated software platforms vastly increased the efficiency of editorial offices allowing multiple journals to be published from a single office or individual journals to be published by an editor and a virtual office. Improved electronic publishing rapidly made the digital format the preferred medium for readers resulting in markedly reduced printing and distribution costs. It was against this background that the open access movement arose with the primary goal of making the results of scientific research free to access, for all and forever.

Although advances in ICT had increased the efficiency of editorial offices and reduced publishing and dissemination costs, some funding was still needed. Therefore if access to scientific information was to be free an alternative to subscription income was required. Article processing or publishing charges (APCs) were thus introduced with the revolutionary idea of switching the cost of access to journal articles from the reader (or often libraries acting as surrogate purchasers) to the author (or often their research funder or author institution). This change in financing was justified by the arguments that the financing of research should include the cost of publication of the research and that the results of publically funded research should be publically and freely available.

A corollary to the advances in ICT and the introduction of APCs was that the financial barrier for new entrants to journal publishing was removed. The advent of cloud computing and specialised open source software programs allowed any entrepreneur with some basic knowledge to start publishing journals with very little capital. At the same time the APCs provided a scalable funding model where costs and expenses were directly related to income received making a journal profitable whether it published a few dozen or a few thousand articles. The result has been an explosion in the number of new journals coming on-line and thousands of new titles appearing with very similar names often confusing both experienced and new researchers.

A consequence of this easy profitability has been that the field of journal publishing has attracted a breed of unscrupulous entrepreneurs more interested in quick profits and less concerned about publication ethics. As journal income is directly related to the number of articles published, there is a clear financial incentive to accept as many articles as possible regardless of quality and to incentivize a kind of vanity publishing. These predatory publishers have tarnished the name of open access publishing and have been used by some critics to cast the whole field of open access publishing into disrepute.

In traditional publishing, institutional journals were often considered as inferior to those journals published by academic societies and commercial publishers. It was often thought that these journals favoured in-house authors, their reviewers were biased against external researchers and in general the journals were too inbred and anachronistic in the modern world of international science. However the same trends that have revolutionised academic publishing have also transformed the best of these journals. ICT has facilitated the opening of these journals with international editorial boards and an international reach attracting authors and readers from many countries. For example, the Memórias do Instituto Oswaldo Cruz has an international editorial board. During the past two years it has received submissions from authors in 70 countries and published articles with authors in 40 countries. The journal since its founding more than a hundred years ago (its first issue was in 1909) has always enjoyed strong institutional support. This has allowed the journal to be freely accessible online from the earliest days of internet journal publishing (since 1994). It has never charged APCs as the Oswaldo Cruz Institute of Oswaldo Cruz Foundation has always believed that the publication of high quality scientific research is an important part of its mandate. This strong institutional support has allowed the journal to concentrate on quality rather than be concerned with income. As a result the Memórias has achieved one of the highest impact factors of any journal in Latin America and is the most cited journal on the continent. More recently the Memórias has adopted the Creative Commons licence and is fully open access compliant. 
The principles of open access are supported by most scholars. They have a professional interest in the widest access to their published work. The moral case for free access to publicly funded research has been eloquently made. However APCs represent a barrier for some researchers who do not have access to funds for publishing, besides the perceived conflict of interest of APCs for journals.

Journals published by academic institutions often do not charge for access and with modern technology those that do, could and should make access free as part of their institutional mandates. These journals, free of the conflict of interest in charging their authors publishing fees, can strive for the highest publishing standards in an open access environment. Of course there are other institutional journals who both provide open access and are leaders in their fields, for example the Bulletin of the World Health Organization, published by World Health Organization in the field of public health, and Emerging Infectious Diseases, published by the Centres for Disease Control. The Memórias is therefore not an anomaly but a pioneer in the re-emergence of institutional journals as an alternative model for open access publishing. 